\title{
DÉFICITS COGNITIVOS Y ABORDAJES TERAPÉUTICOS EN PARÁLISIS CEREBRAL INFANTIL COGNITIVE DEFICITS AND THERAPEUTIC APPROACHES IN CHILDREN WITH CEREBRAL PALSY
}

\author{
Vega Muriel ${ }^{1,2,3}$, Antonia Ensenyat ${ }^{1,2,3}$, Alberto García-Molina ${ }^{1,2,3}$, Celeste \\ Aparicio-López ${ }^{1,2,3}$ Y Teresa Roig-Rovira ${ }^{1,2,3}$.
}

Cómo referenciar este artículo/How to reference this article:

Muriel, V., Ensenyat, A., García-Molina, A., Aparicio-López, C. y Roig-Rovira (2014). Déficits cognitivos y abordajes terapéuticos en parálisis cerebral infantil [Cognitive deficits and therapeutic approaches in children with cerebral palsy]. Acción Psicológica, 11(1), 107-120. http://dx.doi.org/10.5944/ ap.1.1.13915

\section{Resumen}

Objetivo: La parálisis cerebral infantil (PC) está asociada a alteraciones en las capacidades visoperceptivas, déficits atencionales y disfunción ejecutiva. Conocer su repercusión sobre la capacidad funcional y posibles planes terapéuticos, ayuda a mejorar su calidad de vida. Método: Se ha realizado una búsqueda en la literatura sobre la definición, clasificación, perfiles cognitivos y especialmente sobre la estimulación cognitiva en niños con PC. Resultados: Estimular el desarrollo cognitivo para mejorar la calidad de vida, conducta y funcionalidad de estos niños puede dar lugar a cambios en la neuroplasticidad. La integración de estas técnicas, intervención en el habla y tratamiento fisioterapéutico puede mejorar la cognición y motivación en niños con PC. Conclusiones: Los niños con PC y déficits cognitivos se pueden beneficiar de la estimulación cognitiva para mejorar su calidad de vida. Los profesionales clínicos deben brindar una intervención terapéutica centrada en el niño con PC e incluir, la escuela y el entorno familiar.

Palabras clave: Parálisis cerebral; neuropsicología; abordajes terapéuticos.

Abstract
Aim: Cerebral palsy (CP) in children can be as-
sociated with attention deficits, executive dys-
function or alterations in visuoperceptive abili-
ties. Understanding these deficits and possible
therapeutic approaches may improve their
quality of life. Methods: In this study, review
has been carried out into literature about defi-
nition, classification, cognitive profiles and,

1 Institut Guttmann, Institut Universitari de Neurorehabilitació, affiliated with Universitat Autònoma de Barcelona, 08916 Badalona (Barcelona, Spain).

2 Fundació Institut d'Investigació en Ciències de la Salut Germans Trias i Pujol, Badalona (Barcelona, Spain).

3 Departament de Psicología Clínica i de la Salut, Universitat Autònoma de Barcelona, 08193 Bellaterra (Cerdanyola del Vallès, Spain). 
specially, cognitive stimulation of children with $\mathrm{CP}$, given the limited number of publications on this aspect of neurorehabilitation. Results: Stimulating cognitive development in order to improve quality of life, behaviour and functionality of these children may lead to changes in neuroplasticity. Technique integration, coupled with speech intervention and physiotherapeutic treatment can improve cognition and motivation in children with $\mathrm{CP}$ at family and school levels. Conclusions: Children affected by $\mathrm{CP}$ and cognitive deficits can benefit from cognitive stimulation and thus improve their quality of life. Clinicians should offer therapeutic intervention focused on children with $\mathrm{CP}$, while not ignoring school and family environment.

Key words: cerebral palsy; cognitive; neuropsychology; therapeutic approaches.

\section{Introducción}

La parálisis cerebral (PC) es un trastorno del neurodesarrollo atribuido a diferentes momentos del desarrollo cerebral con una prevalencia de 2/1000 niños nacidos, siendo la causa más común de discapacidad física y cognitiva en la edad temprana Los avances en la medicina neonatal han conseguido que sobrevivan más niños, lo que ha dado lugar a un incremento en la prevalencia de la PC (KrägelohMann y Cans, 2009).

La primera referencia en la literatura sobre este trastorno se atribuye al médico británico Willian Little. En 1860, asoció la asfixia durante el parto con la aparición de lesiones permanentes en el sistema nervioso; por ello durante muchos años, este trastorno fue llamado «Enfermedad de Little» (Little, 1861). McKeith, MacKenzie, y Polani, en una carta a la revista Lancet, definieron la PC como un trastorno motor persistente que aparece antes de los tres años debido a interferencias no progresivas en el desarrollo cerebral y que tiene lugar antes de que el crecimiento del sistema nervioso central se complete (McKeith, MacKenzie y Polani, 1959). Hasta hace pocos años, una de las definiciones más utilizadas era la propuesta por Bax en 1964, en la cual se definía la PC como un desorden del movimiento y la postura, ambas por un defecto o una lesión en un cerebro inmaduro (Bax, 1964). Unas décadas después, Mutch y colaboradores definieron la PC como un "término paraguas» por la gran cantidad de síndromes motores, cognitivos y etiologías que hasta la fecha se englobaban en esta patología (Mutch, Alberman, Hagberg, Kodama y Perat, 1992), introduciendo así, la idea de un trastorno a menudo cambiante y no sólo no permanente, como mantenían Mac Keith y Polani.

Ante la variedad de definiciones y clasificaciones utilizadas por los clínicos, un grupo constituido por 14 centros de 8 países formaron el "Surveillance of Cerebral Palsy in Europe». Este grupo de epidemiólogos y clínicos definieron la PC como un conjunto de trastornos permanentes, no cambiantes, del movimiento, postura y función motora que se debe a una lesión, anomalía o interferencia no progresiva de un cerebro en desarrollo o inmaduro (Surveillance of Cerebral Palsy in Europe, 2000). Clasificaron la PC en los siguientes grupos: parálisis cerebral espástica, discinética (distónica o coreo-atetósica) y atáxica.

La PC espástica bilateral es aquella PC en la que existe un aumento del tono muscular de manera persistente en una o varias extremidades y se manifiesta en ambos lados del cuerpo. La PC espástica unilateral es aquella en la que existe un aumento del tono muscular en una o varias extremidades sin embargo, sólo se ve envuelta una parte del cuerpo. En la PC discinética se produce un cambio en el tono muscular; cuando esto ocurre podemos hablar de PC discinética distónica si existe un incremento de este y PC discinética coreoatetósica si el cambio en el tono muscular es de manera decreciente. Por último hablamos de PC atáxica cuando existe una hipotonía generalizada con signos de ataxia (Surveillance of Cerebral Palsy in Europe, 2000).

En 2005, el "Executive Committee for the Definition of Cerebral Palsy" definen la PC como un grupo de desórdenes del desarrollo del movimiento y la postura, que causa limitaciones en las actividades, atribuibles a altera- 
ciones no-progresivas que ocurren en el desarrollo fetal o cerebral del niño. Asimismo, indican que estos desórdenes motores a menudo están acompañados de déficits sensitivos, cognitivos, de comunicación, percepción, y/o comportamiento, y/o crisis comiciales (Bax et al., 2005).

Esta definición introduce conceptos muy valiosos para la práctica clínica neuropsicológica; indica que estos niños pueden presentar déficits cognitivos, comportamentales y de la comunicación. Hasta esa fecha la PC se contemplaba únicamente como un trastorno motor, a pesar de las evidencias de limitaciones que a menudo comportaban problemas funcionales en el entorno, con sus iguales, a nivel educativo y sobre todo, a nivel familiar.

El «Executive Committee for the Definition of Cerebral Palsy» clasifica la PC en función de unos parámetros fundamentales que abarcan la naturaleza de la etiología, déficits asociados, hallazgos anatómicos, radiológicos, las causas y edad de las lesiones (Bax et al., 2005). La naturaleza y tipológica del trastorno motor hace referencia al tipo de anormalidades del tono muscular en reposo o a los trastornos del movimiento en $\mathrm{PC}$.

Los déficits asociados hacen referencia al grado en el que un individuo tiene limitaciones en función del trastorno motor según las parte del cuerpo que se encuentren comprometidas. La presencia o ausencia de problemas no motores en el desarrollo neurológico o sensorial del niño, tales como pueden ser las crisis comiciales, los déficits de audición o visión, los déficits atencionales, comportamentales, de la comunicación en general y los déficits cognitivos se tuvieron en cuenta a la hora de clasificar la PC.

Otra de las claves para la clasificación de la PC fue la categorización en función de los hallazgos anatómicos y radiológicos. La distribución anatómica tiene en cuenta las partes del cuerpo afectadas mientras que los hallazgos radiológicos hacen referencia a los hallazgos neuroanatómicos obtenidos mediante tomografía axial computerizada (TAC) o resonancia magnética (RM), tales como pueden ser la dilatación ventricular, la pérdida de sustancia blanca u otras anormalidades cerebrales. Por último tuvieron en cuenta las causas y el tiempo o marco temporal en el que se produzco el daño.

A la hora de comprender este trastorno hay que tener en cuenta la severidad de la lesión cerebral, el momento vital en el que se produce y el entorno en el que se va a desarrollar el niño. Todos ellos interrelacionan e influyen en los resultados cognitivos y en la capacidad de neuroplasticidad en niños con lesiones congénitas. La edad en la que ocurre el evento es relevante, ya que el nivel de maduración cerebral puede influir en el grado de especialización hemisférica y en la plasticidad además de en el desarrollo funcional de áreas corticales en las diferentes etapas postnatales (Anderson, Spencer-Smith, y Wood, 2011).

\section{Déficits cognitivos en parálisis cerebral}

Durante varias décadas la afectación cognitiva en niños y adultos con PC se ha situado en un segundo plano. La mayoría de estudios se han centrado en investigar las alteraciones físicas, dejando a un lado los aspectos cognitivos que determinan, de igual manera, la calidad de vida de estos pacientes (Pirila et al., 2004). Es en los últimos años cuando han aparecido estudios que sostienen que un tercio de los niños con PC espástica presentan déficits cognitivos, siendo esta proporción de dos tercios en niños con PC de tipo hemipléjica (Rai et al., 2013).

Los niños con PC son especialmente vulnerables a alteraciones en el aprendizaje y disfunción ejecutiva (Bottcher, 2010). Los déficits observados se localizan en las escalas manipulativas penalizadas por los déficits motores y visuales de esta población, por lo que obtienen puntuaciones mejores en escalas verbales incluso con los déficits de comunicación que algunos poseen (Pirila et al., 2004).

Existen déficits del funcionamiento ejecutivo y de la memoria de trabajo en comparación con niños sanos (Baron, Kerns, Müller, Ahronovich y Litman, 2012). Algunos estudios han encontrado diferencias en las medidas de inhibición de respuesta (Lemay, Lê y Lamarre, 2012) 
además de riesgo de déficits en procesos como el lenguaje, percepción visual y memoria (Sigurdardottir et al., 2008). Así mismo estos niños presentan un $2.65 \%$ más de riesgo de desarrollar TDAH durante la edad escolar (Bhutta, Cleves, Casey, Cradock y Anand, 2002).

La respuesta manual es más lenta en pacientes con PC que en controles de su edad, por lo que la evaluación de las funciones cognitivas puede verse afectadas por la presencia de impedimentos motores (Sigurdardottir et al., 2008). Las disfunciones motoras pueden influir en los resultados de los índices manipulativos de las baterías, así como los déficits visuoperceptivos y visoconstructivos, déficits visuales que padecen un alto porcentaje de estos niños y pueden dar lugar a resultados sesgados que necesitan de una evaluación más específica, donde el tiempo y la velocidad de procesamiento de la información estén adaptados.

\section{Atención}

Los déficits de atención sostenida pueden dificultar gravemente la vida diaria de las personas con PC (Lemay et al., 2012). Los déficits atencionales y el trastorno por déficits de atención son unos de los diagnósticos más frecuentes en población con PC (Odding et al., 2006). Pirila et al. (2010) encontraron que jóvenes con PC tenían bajas puntuaciones en pruebas de atención dividida, reducción de la atención requerida para el control postural y respuestas deficitarias de inhibición Este equipo además demostró que la mitad de los participantes jóvenes con PC espástica tenían problemas de atención en el ámbito clínico, especialmente en aquellos con diplejía, en comparación con los sujetos hemipléjicos (Pirila y van der Meere, 2010). En 2006 otro estudio, con una muestra de 15 niños con diplejía espástica, demostró déficits de atención y funcionamiento ejecutivo, evaluados mediante tareas visuales y auditivas (Odding et al., 2006).

Lemay et al. (2012) con una muestra de 10 niños con PC espástica encontraron que estos pacientes realizaban más omisiones en un tiempo de respuesta más variable que el grupo control. Concluyeron que la atención sostenida y la capacidad de inhibición se ven afectados en PC. Kolk y Talvik (2000) y Pirila et al. (2004) utilizaron la batería "A Developmental Neuropsychological Assessment» (NEPSY) para medir la atención, más recientemente Bottcher et al. (2010) usaron la batería de pruebas «Test of Everyday Attention for Children» (TEA-Ch) para medir también la atención con este tipo de población (Bottcher, 2010; Kolk y Talvik, 2000; Pirila et al., 2004). Sin embargo la evaluación utilizada en estos estudios puede verse comprometida por el requerimiento de la memoria y el uso de estímulos auditivos, ambos, posibles déficits de este trastorno (Lemay et al., 2012).

El control postural y sus interferencias en tareas atencionales también han sido objeto de estudio. Reilly et al., (2008) encontraron, en una población entre los 4 y 6 años con PC, un desarrollo deficitario de la atención ejecutiva y el control postural comparado con niños mayores y adultos, experimentando interferencia en el control postural cuando la demanda cognitiva atencional de las tareas era realizada en una postura estática (Reilly, Woollacott, van Donkelaar y Saavedra, 2008).

\section{Lenguaje}

Los niños con PC a menudo han demostrado dificultades en la comunicación y el lenguaje, dependiente en muchos casos, del nivel motor, intelectual y sensorial (Pirila et al., 2007). Pirila et al. (2007) encontraron correlación entre el CI, el lenguaje y el habla. Los niños con un CI por encima de 70 tenían dificultades en el habla es decir, déficits a nivel motor, sin embargo los niños con un CI por debajo de 70 tenían deficiencias en la comprensión, expresión verbal y habilidades motoras del habla. Otro estudio que sostiene la existencia de déficits en el habla y el lenguaje es el de Norderg et al. (2013) que estimaron que existen trastornos del habla en un $21 \%$ de los niños con PC de los cuales un $41 \%$ tenían discapacidad intelectual (Nordberg, Miniscalco, Lohmander y Himmelmann, 2013). 
Cabe destacar sin embargo, que otros estudios en los que se ha evaluado el lenguaje en niños con PC han encontrado relativamente intacta esta capacidad. Estudios como el de Pirila et al. (2004) evaluaron las capacidades cognitivas en niños con diplejía espástica. Hallaron que el lenguaje, junto con la memoria y la capacidad de aprendizaje estaban intactas. Otros estudios corroboran esta afirmación, como el llevado a cabo con una muestra de 127 niños con PC en el que los autores encontraron intactas las habilidades verbales (Sigurdardottir et al., 2008).

Hay estudios que han tenido en cuenta el papel del lenguaje en la aparición de trastornos psiquiátricos en la infancia. Bjorgaas, Hysing y Elgen, (2012) encontraron relación entre los problemas de comunicación en niños con PC y la presencia de trastornos psiquiátricos. Voorman y colaboradores encontraron una asociación entre los problemas de comunicación y problemas de conducta, los cuales, empeoran con el tiempo en los niños con PC (Voorman, Dallmeijer, Van Eck, Schuengel y Becher, 2010); sin embargo, falta investigar si los trastornos psiquiátricos se originan a partir de la lesión cerebral en sí, o en relación a la frustración debido al deterioro de la comunicación lo que puede indicar la necesidad de un mayor énfasis en la intervención temprana para mejorar las habilidades de comunicación en estos niños (Bjorgaas et al., 2012).

\section{Memoria}

Numerosos son los estudios que han hallado déficits en memoria y aprendizaje en los niños con PC (Christ, White, Brunstrom y Abrams, 2003; Gagliardi, Tavano, Turconi y Borgatti, 2013). Østensjø et al. (2003), en una muestra de niños con diferentes subtipos de PC, encontraron que el $44 \%$ de ellos experimentaban dificultades de aprendizaje, en la aplicación de conocimientos en su día a día y déficits de memoria, así mismo, Kolk y Talvik (2000) estudiaron a niños con hemiplejía espástica y los compararon con un grupo control, encontrando diferencias significativas entre ambos grupos (Kolk y Talvik, 2000; Ostensjø, Carlberg y Vøllestad, 2003).

En 2009, Peeters, Verhoeven y Moor, realizaron un estudio con una muestra de 52 niños con PC con trastornos del habla para medir la correlación que existía entre la memoria de trabajo y dichos trastornos (Peeters, Verhoeven y de Moor, 2009). Encontraron que la memoria de trabajo era el mejor predictor de inteligencia, percepción auditiva y habilidades del habla, además de que los niños con PC, déficits intelectuales y déficits del habla tenían un mayor riesgo de resultados bajos en memoria de trabajo verbal.

\section{Visopercepción/visoconstrucción}

Las capacidades visuoperceptivas y visoconstructivas han sido las funciones cognitivas más estudiadas en población infantil con PC. Goto et al. (1994) encontraron que el $72 \%$ de los sujetos prematuros con diplejía espástica presentaban déficits visuoespeciales y visoconstructivos obtenidos en los subtests de cubos y el 70\% tenían déficits visuoespaciales mediante la evaluación del subtest de figuras incompletas, ambos subtest de la Escala de inteligencia de Weschler para niños (Goto, Ota, Iai, Sugita y Tanabe, 1994).

En un estudio Pagliano et al., (2007) encontró correlación entre la severidad de la leucomalacia periventricular y los déficits en las funciones visoperceptivas. Evaluaron 15 niños prematuros y nueve niños nacidos a término con diplejía espástica y leucomalacia periventricular. Las puntuaciones obtenidas por el grupo prematuro fueron inferiores a lo normal, encontrando afectadas las habilidades visuoperceptiva (Pagliano et al., 2007).

Varios estudios han asociado el deterioro visuoperceptivo con la reducción de la sustancia blanca en el lóbulo parietal y lóbulo occipital en niños con PC espástica (Goto et al., 1994; Rai et al., 2013). Coinciden en la idea de que una pérdida de sustancia blanca en el lóbulo parietal se relaciona con un peor rendimiento en las capacidades visuoperceptivas y visoconstructivas, así como una reducción en 
la sustancia blanca occipital se relaciona con el rendimiento en la capacidad visoconstrucitvas (Goto et al., 1994).

\section{Funcionamiento ejecutivo}

La PC se asocia con déficits en el sistema ejecutivo (Bottcher, 2010; Pirila y van der Meere, 2010). En la práctica clínica los niños con PC, destacan por déficits de rendimiento en el control inhibitorio y flexibilidad cognitiva, lo que ayuda a explicar la relación con manifestaciones conductuales, problemas sociales y de aprendizaje (Bodimeade, Whittingham, Lloyd y Boyd, 2013; Bottcher, 2010). Sin embargo en el estudio de Lemay et al. (2012) el número de comisiones observadas, en la prueba Continuous Performance Test (CPT), entre un grupo control y un grupo de 10 niños con PC fue similar, por lo que no observaron déficits en la inhibición.

Se ha encontrado un perfil disejecutivo propio de los niños con PC presente durante todo el desarrollo temprano, caracterizado por problemas de atención combinado con déficits de impulsividad. En un estudio de Pirila et al. (2011) los niños con las lesiones bilaterales obtuvieron peores puntuaciones que los niños con lesiones unilaterales, además evaluaron la relación entre las semanas de embarazo y la disfunción ejecutiva, encontrando correlación como planteaba Plagiano et al. (2007). En un estudio de niños con hemiplejia espástica, Kolk y Talvik (2000) estudiaron la inhibición de los impulsos y encontraron que los niños hemipléjicos, especialmente aquellos con lesiones del hemisferio derecho, puntuaron significativamente más bajo que el grupo control. Crist et al., (2003) estudiaron el control inhibitorio en niños con PC espástica bilateral encontrando alteración de esta capacidad en comparación con un grupo control sano. La diferencia entre este estudio y el de Lemay et al. (2012) puede ser debido al tamaño de la muestra y al requerimiento motor en el CPT en comparación con la prueba de Stroop utilizada por Crist et al. (2003).
En conclusión los efectos combinados de la PC, el hecho de que sean lesiones bilaterales y un parto prematuro correlacionan con problemas del funcionamiento cognitivo. Es necesario recalcar la necesidad de una identificación temprana de los déficits ejecutivos con PC para mejorar el rendimiento académico de los niños, el cual puede ser deficitario por una falta de identificación de los déficits.

\section{Calidad de vida y funcionalidad}

Los déficits visuales, auditivos, crisis comiciales y déficits cognitivos interfieren con las habilidades para un buen funcionamiento en la calidad de vida de los niños con PC. Los déficits motores interfieren en el día a día y los déficits neuropsicológicos suponen, para los pacientes y sus familiares, una carga de la enfermedad tan importante como los primeros (Legault, Shevell y Dagenais, 2011).

Los niños con trastornos del neurodesarrollo y déficits cognitivos tienen un mayor riesgo de tener dificultades en el ámbito social. Se ha encontrado que estos presentan mayores dificultades en las interacciones sociales que niños sanos; además los problemas conductuales son cinco veces más probables en comparación a sus iguales (Odding et al., 2006). Alrededor de una cuarta parte de los niños con PC manifiesta síntomas emocionales y de comportamiento, siendo los problemas más comunes las habilidades sociales con sus iguales, la hiperactividad, las alteraciones emocionales, labilidad emocional, irritabilidad, impulsividad, déficits de atención y problemas en la toma de decisiones, con todo el efecto, en el núcleo familiar, que esto supone (Parkes et al., 2008). Hay estudios que han encontrado relación entre el funcionamiento cognitivo y motor (Enkelaar, Ketelaar, y Gorter, 2008). Sin embargo hay estudios como el de Chong et al. (2012) donde no se encuentra relación entre la satisfacción de vida en un grupo de 48 niños con PC y sus habilidades funcionales como puede pensarse, sino con la percepción que estos mismos tienen del trastorno (Chong, Mackey, Broadbent y Stott, 2012). Evaluaron la percepción de la calidad de vida de niños con PC encontrando que no exis- 
tía relación entre la edad, la capacidad funcional y la percepción de satisfacción. Las puntuaciones de bienestar y salud autopercibida están influenciadas por el dolor y el posible deterioro de las funciones, pero no por las capacidades cognitivas (Furukawa, Iwatsuki, Nishiyama, Nii y Uchida, 2001).

La PC es una de las condiciones neurológicas con un mayor riesgo asociado a problemas de salud (Parkes et al., 2008; Sigurdardottir et al., 2008). Bjorgaas, Hysing y Elgen evaluaron un cohorte de 67 niños entre 2001 y 2003 para estudiar la prevalencia de trastornos psiquiátricos en niños con PC mediante un cuestionario administrado a los padres (Kidie-SADS). Encontraron que el 57\% de los niños tenían trastornos psiquiátricos, 28 de ellos cumplían los criterios de TDAH, el más común de todos los trastornos psiquiátricos. Los niños con trastornos de comunicación eran más proclives a desarrollar dichos trastornos psiquiátricos; sin embargo no se encontraron diferencias significativas según el tipo de PC (Bjorgaas et al., 2012).

Una atención temprana, tratamiento neuropsicológico del menor y una intervención familiar para lidiar con las dificultades diarias de este trastorno ayudaría a reducir el estrés de los cuidadores y del propio niño (Majnemer, Shevell, Law, Poulin, y Rosenbaum, 2012). La implicación del centro escolar y de los profesores, así como la integración del niño en actividades que potencien sus habilidades sociales, puede suponer una mejora en su percepción de satisfacción.

\section{Plasticidad y abordajes terapéuticos}

Las repercusiones que pueden tener las lesiones cerebrales en el PC, determinan los déficits neuropsicológicos asociados, así como la calidad de vida de los niños y se deben principalmente a dos factores: el lugar anatómico de la lesión y el momento vital en el que se produce. El lugar de la lesión es un factor importante predictivo del perfil de deterioro cognitivo. Lesiones en el hemisferio izquierdo están asociadas a un deterioro significativo en el lenguaje y lesiones en el hemisferio derecho están asociadas a deterioro de la atención y visopercepción (Kolk y Talvik, 2000). Una intervención temprana en niños con PC podría dar lugar a una mejora en la reorganización cerebral y ayudar con ello, de manera directa, a la calidad de vida de estos pacientes. La distribución de las lesiones ha recibido mucha atención por parte de clínicos e investigadores que han centrado sus estudios en la reorganización interhemisférica del lenguaje o la lateralización de las lesiones en sujetos hemipléjicos. Se ha encontrado que los déficits en escalas manipulativas no son específicos de sujetos con hemiplejia izquierda y lesiones en el hemisferio derecho sino que también se han observado en sujetos con hemiplejia derecha con lesiones del hemisferio izquierdo (Goodman y Yude, 1996). En este contexto apareció el denominado efecto de saturación (crowding hypothesis). Según este, los niños con lesiones del hemisferio izquierdo preservan el lenguaje lateralizando estas funciones en el hemisferio derecho, aunque esto vaya en detrimento de las funciones comúnmente asociadas a este hemisferio como son las capacidades visoperceptivas (Karen Lidzba, Staudt, Wilke y Krägeloh-Mann, 2006). Esta hipótesis ha sido confirmada en varios estudios realizados en niños con lesiones cerebrales congénitas (Guzzetta et al., 2008), es decir, en un cerebro inmaduro el hemisferio derecho podría asumir funciones propias del lenguaje comúnmente asociado al hemisferio izquierdo (Hertz-Pannier et al., 2002). Estudios como el de Lidzba demostraron que el lenguaje se puede reorganizar en áreas homólogas del hemisferio derecho después de lesiones tempranas del hemisferio izquierdo (Lidzba, Wilke, Staudt, Krägeloh-Mann y Grodd, 2008).

El momento vital en el que se produce la lesión es un factor a tener en cuenta al valorar las repercusiones generadas por la PC. Los resultados en los estudios neuropsicológicos son contradictorios, unos autores consideran que existe una relación entre la edad y las posibles consecuencias que pueden darse en el desarrollo de futuros déficits; es decir, consideran que cuanto más joven es el niño peor pronóstico a nivel cognitivo (Max, 2004). Otros consideran 
sin embargo lo contrario (Westmacott, Askalan, MacGregor, Anderson y Deveber, 2010). Esto puede deberse a la hipótesis de que las lesiones cerebrales tempranas obtienen resultados más satisfactorios por los procesos de neuroplasticidad (Riva et al., 2013). Estas afirmaciones sin embargo han sido refutadas por otros estudios (Westmacott et al., 2010).

El principio de Kennard señala que existe una relación líneal negativa entre la edad en la que se produce la lesión cerebral y el resultado funcional de esta en igualdad de circunstancias, es decir, cuanto más joven sea el organismo lesionado, mejor será el resultado (Dennis, 2010). Otros autores, como Kolb et al. 2010 sostienen que la capacidad funcional de recuperación no está relacionada inversamente con la edad de la lesión sino que alcanza un nivel óptimo cuando la etapa de desarrollo y maduración es más favorable para la sinaptogénesis y la formación glial (Bryan Kolb, Teskey y Gibb, 2010).

Hay escasas publicaciones sobre estimulación cognitiva en pacientes con PC. El objetivo del tratamiento rehabilitador se ha desplazado a la rehabilitación neurológica en respuesta a la creciente evidencia de la neuroplasticidad (Aisen et al., 2011). El cerebro está en desarrollo durante la etapa fetal, postnatal y pasada la primera década, responsable del moldeado por experiencia.

Aisen et al. (2011) proponen la necesidad de establecer una rehabilitación cognitiva en el caso de que existieran alteraciones cognitivas. Para ello proponen una intervención neuropsicológica, del habla y una evaluación que de buena cuenta de las alteraciones y la naturaleza de los déficits (si por ejemplo se trata de déficits de atención y no de memoria), así como la consideración farmacológica para los déficits de atención y cognición si el facultativo lo considera necesario, intervención con la familia y la escuela para establecer planes educativos individuales y dispositivos de comunicación adecuados (Aisen et al., 2011).

Se han utilizado las nuevas tecnologías, como la realidad virtual, para el abordaje de funciones motoras y cognitivas (Dinomais et al., 2013). Holt et al. (2011) llegaron a la con- clusión de que la exposición a ambientes sensoriales enriquecidos y programas de desarrollo temprano mejoran la función cognitiva y el crecimiento cerebral en niños con daño cerebral perinatal (Holt y Mikati, 2011). Reid y Harris (2005) realizaron una investigación sobre los efectos de la realidad virtual en la percepción de autoeficacia, control y competencia, el control de las extremidades superiores y el control postural. Los niños muestran mejora en el control postural en comparación con el grupo control (Harris y Reid, 2005). En un estudio realizado en 2003, Reid investigó la influencia de la realidad virtual en la percepción de felicidad de niños con PC. Encontró que los entornos virtuales permiten a los niños ser creativos y desafiante siendo una motivación para los participantes. Hay que tener en cuenta que para los niños estar demasiado tiempo delante de una pantalla es un factor causante de inactividad física y que para el uso de estas intervenciones es necesaria una alta capacidad atencional y motivacional, mejorada en algunos estudios, mediante la regulación de la dificultad en tareas según los objetivos terapéuticos y los objetivos a alcanzar por el niño (Wang y Reid, 2011).

Akhutina et al. (2003) realizaron un tratamiento computerizado a 12 niños con PC. Realizaron ejercicios que consistían en un laberinto (una mariquita que tenía que llegar a un árbol) con diferentes niveles de dificultad. Encontraron que el grupo experimental obtenía mejoras en su funcionamiento espacial sin embargo, hubo un grupo de niños cuyo nivel inicial de rendimiento era bajo y no pudo sacar beneficio del tratamiento (Akhutina et al., 2003).

\section{Conclusiones}

En conclusión, ante el aumento de demanda sanitaria por parte de este colectivo y sus familiares, nuestro equipo considera necesario crear líneas de investigación que den cuenta de la importancia de implementar una intervención multidisciplinar donde lo psicológico y neuropsicológico estén incluidos. Potenciar un entorno propicio para el desarrollo de las capacidades cognitivas del niño, la puesta en 
marcha de pautas a nivel escolar y familiar, y una intervención neuropsicológica en esta población, ayudaría a mejorar la visión sobre la satisfacción de vida y funcionalidad de estos niños.

En primer lugar se debería realizar una intervención familiar en la que los padres compartan sus dudas sobre los déficits de los niños con PC en el caso de que existieran, psicoeducación para manejar la discapacidad de los menores así como proporcionar pautas a estos, para mejorar sus habilidades sociales y potenciar las relaciones con sus iguales.

En segundo lugar, desde la intervención psicológica hay que tener en cuenta los posibles trastornos psiquiátricos que este trastorno puede conllevar. Diagnosticarlo a tiempo puede suponer mejoras a nivel académico y por consiguiente mejoras a nivel cognitivo que pueden haber estado enmascaradas. Es necesario que los clínicos y médicos de familia conozcan, en la medida de lo posible, los posibles síntomas psiquiátricos o su derivación a centros especializados y multidisciplinares donde los niños con PC reciban una rehabilitación física y cognitiva que potencie al máximo sus capacidades.

\section{Referencias bibliográficas}

Aisen, M. L., Kerkovich, D., Mast, J., Mulroy, S., Wren, T. A. L., Kay, R. M. y Rethlefsen, S. A. (2011). Cerebral palsy: clinical care and neurological rehabilitation. Lancet neurology, 10(9), 844-852. doi:10.1016/S1474-4422(11)70176-4.

Akhutina, T., Foreman, N., Krichevets, A., Matikka, L., Narhi, V., Pylaeva, N. y Vahakuopus, J. (2003). Improving spatial functioning in children with cerebral palsy using computerized and traditional game tasks. Disability and rehabilitation, 25(24), 1361-1371. doi:10.1080/09638 280310001616358.

Anderson, V., Spencer-Smith, M. y Wood, A. (2011). Do children really recover better? Neurobehavioural plasticity after early brain insult. Brain: a journal of neurology, 134(8), 2197-2221. doi:10.1093/brain/awr103.
Baron, I. S., Kerns, K. A., Müller, U., Ahronovich, M. D. y Litman, F. R. (2012). Executive functions in extremely low birth weight and late-preterm preschoolers: effects on working memory and response inhibition. Child neuropsychology: a journal on normal and abnormal development in childhood and adolescence, 18(6), 586-599. do i:10.1080/09297049.2011.631906.

Bax, M. C. (1964). Terminology and classification of cerebral palsy. Developmental medicine and child neurology, 6, 295-297.

Bax, M., Goldstein, M., Rosenbaum, P., Leviton, A., Paneth, N., Dan, B. y Damiano, D. (2005). Proposed definition and classification of cerebral palsy, April 2005. Developmental Medicine y Child Neurology, 47(8), 571-576.

Bjorgaas, H. M., Hysing, M. y Elgen, I. (2012). Psychiatric disorders among children with cerebral palsy at school starting age. Research in developmental disabilities, 33(4), 1287-1293. doi:10.1016/j.ridd.2012.02.024.

Bodimeade, H. L., Whittingham, K., Lloyd, O. y Boyd, R. N. (2013). Executive functioning in children with unilateral cerebral palsy: protocol for a cross-sectional study. BMJ open, 3(4). doi:10.1136/bmjopen-2012-002500.

Bottcher, L. (2010). Children with Spastic Cerebral Palsy, Their Cognitive Functioning, and Social Participation: A Review. Child Neuropsychology, 16(3), 209-228. doi:10.1080/09297040903559630.

Bhutta, A. T., Cleves, M. A., Casey, P. H., Cradock, M. M. y Anand, K. J. S. (2002). Cognitive and behavioral outcomes of school-aged children who were born preterm: a meta-analysis. JAMA: the journal of the American Medical Association, 288(6), 728-737.

Chong, J., Mackey, A. H., Broadbent, E. y Stott, N. S. (2012). Children's perceptions of their cerebral palsy and their impact on life satisfaction. Disability and rehabilitation, 34(24), 2053-2060. doi:10.3109/09638288.2012.669021

Christ, S., White, D., Brunstrom, J. E. y Abrams, R. (2003). Inhibitory control following perinatal brain injury. Neuropsychology, 17, 171-178.

Dennis, M. (2010). Margaret Kennard (1899-1975): not a "principle» of brain plasticity but a founding mother of developmental neuropsycholo- 
gy. Cortex; a journal devoted to the study of the nervous system and behavior, 46(8), 1043-1059. doi:10.1016/j.cortex.2009.10.008.

Dinomais, M., Veaux, F., Yamaguchi, T., Richard, P., Richard, I. y Nguyen, S. (2013). A new virtual reality tool for unilateral cerebral palsy rehabilitation: Two single-case studies. Developmental neurorehabilitation. doi:10.3109/17518423.201 3.778347 .

Furukawa, A., Iwatsuki, H., Nishiyama, M., Nii, E. y Uchida, A. (2001). Study on the subjective well-being of adult patients with cerebral palsy. Journal of physical therapy science, 13, 31-35.

Gagliardi, C., Tavano, A., Turconi, A. C. y Borgatti, R. (2013). Sequence memory skills in Spastic Bilateral Cerebral Palsy are age independent as in normally developing children. Disability and rehabilitation, 35(6), 506-512. doi:10.3109/0963 8288.2012.704124.

Goodman, R., y Yude, C. (1996). IQ and its predictors in childhood hemiplegia. Developmental medicine and child neurology, 38(10), 881-890.

Goto, M., Ota, R., Iai, M., Sugita, K. y Tanabe, Y. (1994). MRI changes and deficits of higher brain functions in preterm diplegia. Acta paediatrica (Oslo, Norway: 1992), 83(5), 506-511.

Guzzetta, A., Pecini, C., Biagi, L., Tosetti, M., Brizzolara, D., Chilosi, A. y Cioni, G. (2008). Language organisation in left perinatal stroke. Neuropediatrics, 39(3), 157-163. doi:10.1055/s-0028-1085465.

Harris, K. y Reid, D. (2005). The influence of virtual reality play on children's motivation. Canadian journal of occupational therapy. Revue canadienne d'ergothérapie, 72(1), 21-29.

Kolb, Bryan, Teskey, G. C. y Gibb, R. (2010). Factors influencing cerebral plasticity in the normal and injured brain. Frontiers in human neuroscience, 4, 204. doi:10.3389/fnhum.2010.00204.

Kolk, A. y Talvik, T. (2000). Cognitive outcome of children with early-onset hemiparesis. Journal of child neurology, 15(9), 581-587.

Krägeloh-Mann, I. y Cans, C. (2009). Cerebral palsy update. Brain and Development, 31(7), 537-544. doi:10.1016/j.braindev.2009.03.009.

Legault, G., Shevell, M. I., Dagenais, L. y Quebec Cerebral Palsy Registry (Registre de la paralysie cérébrale au Québec [REPACQ]) Consortium. (2011). Predicting comorbidities with neuroimaging in children with cerebral palsy. Pediatric neurology, 45(4), 229-232. doi:10.1016/j.pediatrneurol.2011.06.005.

Lemay, M., Lê, T.-T. y Lamarre, C. (2012). Deficits in two versions of a sustained attention test in adolescents with cerebral palsy. Developmental neurorehabilitation, 15(4), 253-258. doi:10.3109/ 17518423.2012 .678020 .

Little, WJ. (1861). On the influence of abnormal parturition, difficult labours, premature birth, and asphyxia neonatorum, on the mental and physical condition of the child, especially in relation to deformities. The Obstestric Society of London, 3, 293.

Lidzba, K, Wilke, M., Staudt, M., Krägeloh-Mann, I. y Grodd, W. (2008). Reorganization of the cerebro-cerebellar network of language production in patients with congenital left-hemispheric brain lesions. Brain and language, 106(3), 204210. doi:10.1016/j.bandl.2007.11.003.

Majnemer, A., Shevell, M., Law, M., Poulin, C. y Rosenbaum, P. (2012). Indicators of distress in families of children with cerebral palsy. Disability and rehabilitation, 34(14), 1202-1207. doi:10. 3109/09638288.2011.638035.

Max, J. E. (2004). Effect of side of lesion on neuropsychological performance in childhood stroke. Journal of the International Neuropsychological Society: JINS, 10(5), 698-708. doi:10.1017/ S1355617704105092.

McKeith, R., MacKenzie, I. y Polani, P. (1959). Memorandum on terminology and classification of «Cerebral palsy». Cerebral Palsy Bull, 1, 27-35. doi:10.1111/j.1440-1754.2010.01896.x.

Nordberg, A., Miniscalco, C., Lohmander, A. y Himmelmann, K. (2013). Speech problems affect more than one in two children with cerebral palsy: Swedish population-based study. Acta paediatrica (Oslo, Norway: 1992), 102(2), 161166. doi:10.1111/apa.12076.

Odding, E., Roebroeck, M. E, y Stam, H. J. (2006). The epidemiology of cerebral palsy: incidence, impairments and risk factors. Disability and rehabilitation, 28(4), 183-191. doi:10.1080/09638280500158422. 
Ostensjø, S., Carlberg, E. B. y Vøllestad, N. K. (2003). Everyday functioning in young children with cerebral palsy: functional skills, caregiver assistance, and modifications of the environment. Developmental medicine and child neurology, 45(9), 603-612.

Pagliano, E., Fedrizzi, E., Erbetta, A., Bulgheroni, S., Solari, A., Bono, R., iva, D. (2007). Cognitive profiles and visuoperceptual abilities in preterm and term spastic diplegic children with periventricular leukomalacia. Journal of child neurology, 22(3), 282-288. doi:10.1177/0883073807300529.

Peeters, M., Verhoeven, L. y de Moor, J. (2009). Predictors of verbal working memory in children with cerebral palsy. Research in developmental disabilities, 30(6), 1502-1511. doi:10.1016/j. ridd.2009.07.014.

Parkes, J., White-Koning, M., Dickinson, H. O., Thyen, U., Arnaud, C., Beckung, E., Colver, A. (2008). Psychological problems in children with cerebral palsy: a cross-sectional European study. Journal of Child Psychology and Psychiatry, 49(4), 405-413. doi:10.1111/j.14697610.2007.01845.x.

Pirila, S, y van der Meere, J. (2010). Cerebral palsy: effects of early brain injury on development. Handbook of Medical Neuropsychology: Applications of Cognitive Neuroscience. Nueva York: Springer.

Pirila, Silja, van der Meere, J., Korhonen, P., RuusuNiemi, P., Kyntaja, M., Nieminen, P. y Korpela, R. (2004). A retrospective neurocognitive study in children with spastic diplegia. Developmental neuropsychology, 26(3), 679-690. doi:10.1207/ s15326942dn2603_2.

Pirila, Silja, van der Meere, J., Pentikainen, T., Ruusu-Niemi, P., Korpela, R., Kilpinen, J. y Nieminen, P. (2007). Language and motor speech skills in children with cerebral palsy. Journal of communication disorders, 40(2), 116-128. doi:10.1016/j.jcomdis.2006.06.002.

Rai, Y., Chaturvedi, S., Paliwal, V. K., Goyal, P., Chourasia, A., Singh Rathore, R. K., Gupta, R. K. (2013). DTI correlates of cognition in term children with spastic diplegic cerebral palsy. European journal of paediatric neurology: EJPN: official journal of the European Paediatric Neu- rology Society, 17(3), 294-301. doi:10.1016/j. ejpn.2012.11.005.

Reilly, D. S., Woollacott, M. H., van Donkelaar, P. y Saavedra, S. (2008). The Interaction Between Executive Attention and Postural Control in Dual-Task Conditions: Children With Cerebral Palsy. Archives of Physical Medicine and Rehabilitation, 89(5), 834-842. doi:10.1016/j. apmr.2007.10.023

Riva, D., Franceschetti, S., Erbetta, A., Baranello, G., Esposito, S. y Bulgheroni, S. (2013). Congenital brain damage: cognitive development correlates with lesion and electroencephalographic features. Journal of child neurology, 28(4), 446-454. doi:10.1177/0883073812447684.

Sigurdardottir, S., Eiriksdottir, A., Gunnarsdottir, E., Meintema, M., Arnadottir, U. y Vik, T. (2008). Cognitive profile in young Icelandic children with cerebral palsy. Developmental Medicine $y$ Child Neurology, 50(5), 357-362. doi:10.1111/ j.1469-8749.2008.02046.x.

Surveillance of Cerebral Palsy in Europe (2000). Surveillance of cerebral palsy in Europe: a collaboration of cerebral palsy surveys and registers. Surveillance of Cerebral Palsy in Europe (SCPE). Developmental medicine and child neurology, 42(12), 816-824.

Voorman, J. M., Dallmeijer, A. J., Van Eck, M., Schuengel, C. y Becher, J. G. (2010). Social functioning and communication in children with cerebral palsy: association with disease characteristics and personal and environmental factors. Developmental medicine and child neurology, 52(5), 441-447.

Wang, M. y Reid, D. (2011). Virtual Reality in Pediatric Neurorehabilitation: Attention Deficit Hyperactivity Disorder, Autism and Cerebral Palsy. Neuroepidemiology, 36(1), 2-18. doi:10.1159/000320847.

Westmacott, R., Askalan, R., MacGregor, D., Anderson, P. y Deveber, G. (2010). Cognitive outcome following unilateral arterial ischaemic stroke in childhood: effects of age at stroke and lesion location. Developmental medicine and child neurology, 52(4), 386-393. doi:10.1111/j.14698749.2009.03403.x. 


